l'ouverture des frontières, les dépenses de notre pays pour une bonne formation universitaire se justifieront encore politiquement, alors que les frais de l'Etat sont en constante augmentation. On est en droit de craindre, du moins, une lente diminution de la qualité de notre médecine, la faisant rejoindre le niveau moyen européen.
Ces quelques exemples montrent que dans l'opinion publique, les conséquences sérieusement négatives des accords bilatéraux sont largement sousestimées et banalisées, souvent en raison d'une propagande unilatérale de leurs partisans. Dès lors, un engagement pour la vie mérite un sérieux examen préventif!

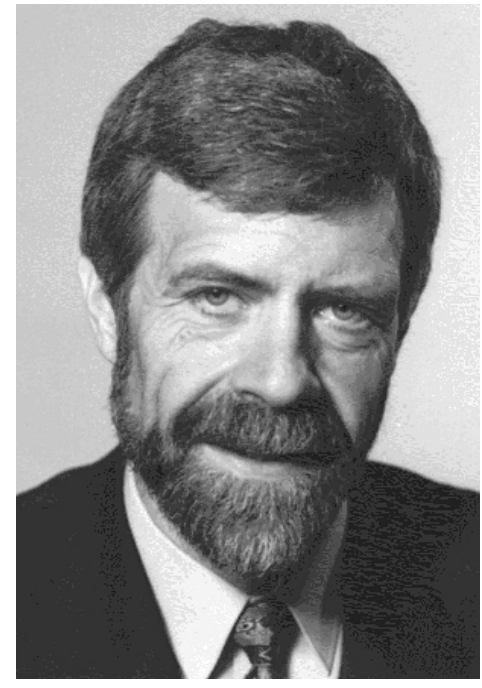

\section{Un pas nécessaire vers l'intégration}

\author{
P. Günter
}

Pour le parti socialiste, il est clair qu'une intégration à l'Europe est nécessaire pour notre pays. Ce processus n'ira pas sans difficultés. Mais tout problème trouve sa solution, particulièrement si l'on songe que la Suisse, grâce à son haut niveau d'éducation et à sa solide infrastructure, est bien placée sur le plan de la concurrence par rapport aux autres nations européennes. Par contre, l'isolement conduirait à la stagnation et, à la longue, à un régression économique et culturelle.

Il va de soi que les conventions impliquent également des devoirs et, en l'occurrence, quelques couleuvres à avaler. Sur le plan des négociations, la Suisse a cependant obtenu des résultats étonnamment favorables: cinq des sept dossiers en discussion sont nettement en faveur de la Suisse (recherche, obstacles techniques au commerce, marchés publics, ouverture du marché aux produits agricoles, transport aérien). On peut dire sans se tromper qu'un rejet des accords bilatéraux avec l'UE signifierait de nouvelles négociations avec des résultats beaucoup moins bons.

\section{Dossier des transports terrestres}

Les marchés routiers et ferroviaires devraient progressivement s'ouvrir. Certes, l'accord devrait effectivement occasionner un afflux de poids lourds sur nos routes. Cela dit, les conventions prévoient des mesures en cas de pléthore de véhicules de gros calibre. Pour éviter l'augmentation des poids lourds, nous avons, comme mesure d'accompagnement, l'article concernant la protection des Alpes, la redevance sur le trafic des poids lourds liée aux prestations (RPLP) et les grands projets inhérents aux transports publics (NLFA, tunnel sous les Alpes).

Qu'arriverait-il en cas de refus? Les événements ont montré jusqu'ici que le rejet existant envers les camions, même sans convention, a diminué sous la pression des pays environnants. Pour les 40 tonnes, le rayon d'action à partir de la frontière s'étend constamment et des exceptions sont faites. C'est ainsi que les 40 tonnes chargés de bois (suite à l'ouragan "Lothar") peuvent bientôt circuler partout. Un rejet des accords bilatéraux aurait pour conséquence de ne pas pouvoir percevoir la RPLP, mettant de surcroît en péril les projets ferroviaires (sauf si l'on perçoit de nouveaux impôts). Résultat: même si l'aval donné au dossier des transports terrestres n'est pas l'idéal pour l'environnement, le rejeter serait plus néfaste encore. 


\section{Dossier sur la libre circulation des personnes}

Une pression accrue de la concurrence pourrait toucher notre profession, surtout à proximité des frontières. La Confédération, s'armant de mesures d'accompagnement, tente d'en atténuer les effets. Ces mesures ont été réclamées avec force, principalement par le PS et les syndicats. Comme on envisage maintenant des mesures efficaces contre le dumping social et salarial, le PS et les syndicats soutiennent également ce dossier.

N'oublions pas que la libre circulation des personnes s'ouvre également à notre corporation, à la relève en particulier, qui pourra exercer dans toute l'Europe.

Une décision spéciale a été prise à l'intention du corps médical, selon laquelle les médecins étrangers s'installant en Suisse devront au moins posséder l'une de nos langues nationales. On ne saurait demander plus sans transgresser les prescriptions légales antidiscriminatoires.
Au demeurant, notons que la situation est largement entre nos mains. Nos médecins-chefs, qui engagent d'ores et déjà des assistants allemands acceptant de gagner moins (avec l'aval des administrations concernées), espèrent déjà pouvoir faire des économies salariales. Il va de soi, cependant, que la plupart d'entre eux peuvent choisir leurs collaborateurs et aider ainsi à contenir un flux exagéré de médecins étrangers.

N'oublions pas non plus qu'il n'est possible d'exercer notre profession au niveau que nous lui connaissons que dans un environnement économique prospère. A cet égard, je suis convaincu de la nécessité des bilatérales.

En conclusion, je ne saurais cacher que, pour le PS, les accords bilatéraux sont un pas important vers l'intégration européenne. Mais il est tout aussi clair que les bilatérales ne permettent pas de préjuger de ce pas.

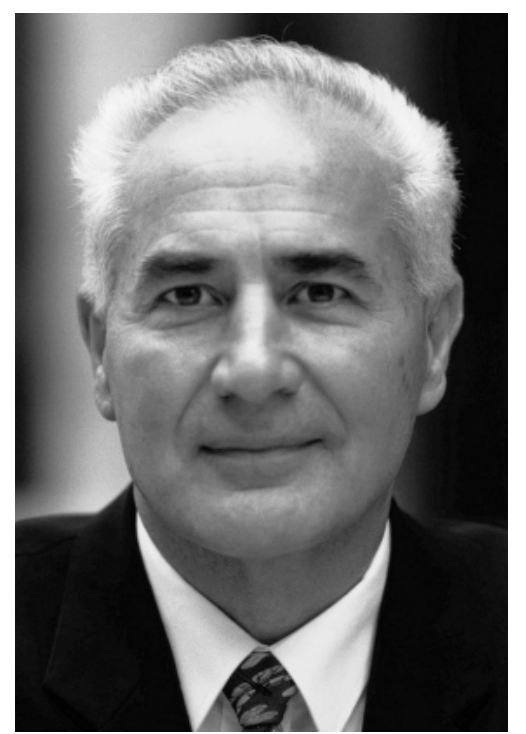

Correspondance:

Dr Guido A. Zäch

Conseiller national PDC

Centre suisse de paraplégiques

CH-6207 Nottwil
Négocier c'est établir les termes d'un échange. Cette assertion s'avère particulièrement vraie en ce qui concerne les très complexes accords bilatéraux qui seront soumis au vote populaire le 21 mai prochain. Du point de vue de la politique de la santé, l'évaluation de ces accords doit donc, elle aussi, porter sur les aspects négatifs et les aspects positifs. Or, ces derniers font manifestement pencher la balance en leur faveur.

Disons d'emblée que les sept accords bilatéraux sont en premier lieu des accords économiques qui devraient avoir pour effet d'améliorer de part et d'autre l'accès au marché intérieur européen. Du point de vue de la politique de la santé, les accords ci-après me paraissent revêtir une importance particulière.

\section{Reconnaissance mutuelle des diplômes}

L'accord sur la libre circulation des personnes prévoit la reconnaissance mutuelle des diplômes pour les médecins, les dentistes, les pharmaciens, le personnel soignant et les sages-femmes. Cette reconnaissance conduira, conjointement à un assouplissement du marché du travail, à un recrutement facilité de personnes qualifiées dans le domaine de la santé. Comme des mesures internes au pays peuvent être prévues pour garantir la qualité des prestations médicales, il n'y a rien à objecter. 